\title{
Risk-Hedged Approach for Re-routing Air Traffic Under Weather Uncertainty
}

\author{
Alexander V. Sadovsky \\ Karl D. Bilimoria
}

NASA Ames Research Center

AIAA Aviation 2016

June 13-17, Washington, D.C. 


\section{Outline}

- Background on route planning

- Re-routing options for weather avoidance

- Risk-hedged approach for re-routing

- Example results

- Conclusion 


\section{Background}

- Flight operators design the routes they wish to fly

- Air traffic service provider designs and implements re-routing around bad weather

- Strategic planning for re-routes around large weather systems is based on multi-hour weather forecasts

- Multi-hour weather forecasts have high uncertainty, but current products typically provide only the most likely instantiation of future weather 


\section{Re-routing for Weather Avoidance}

either, or

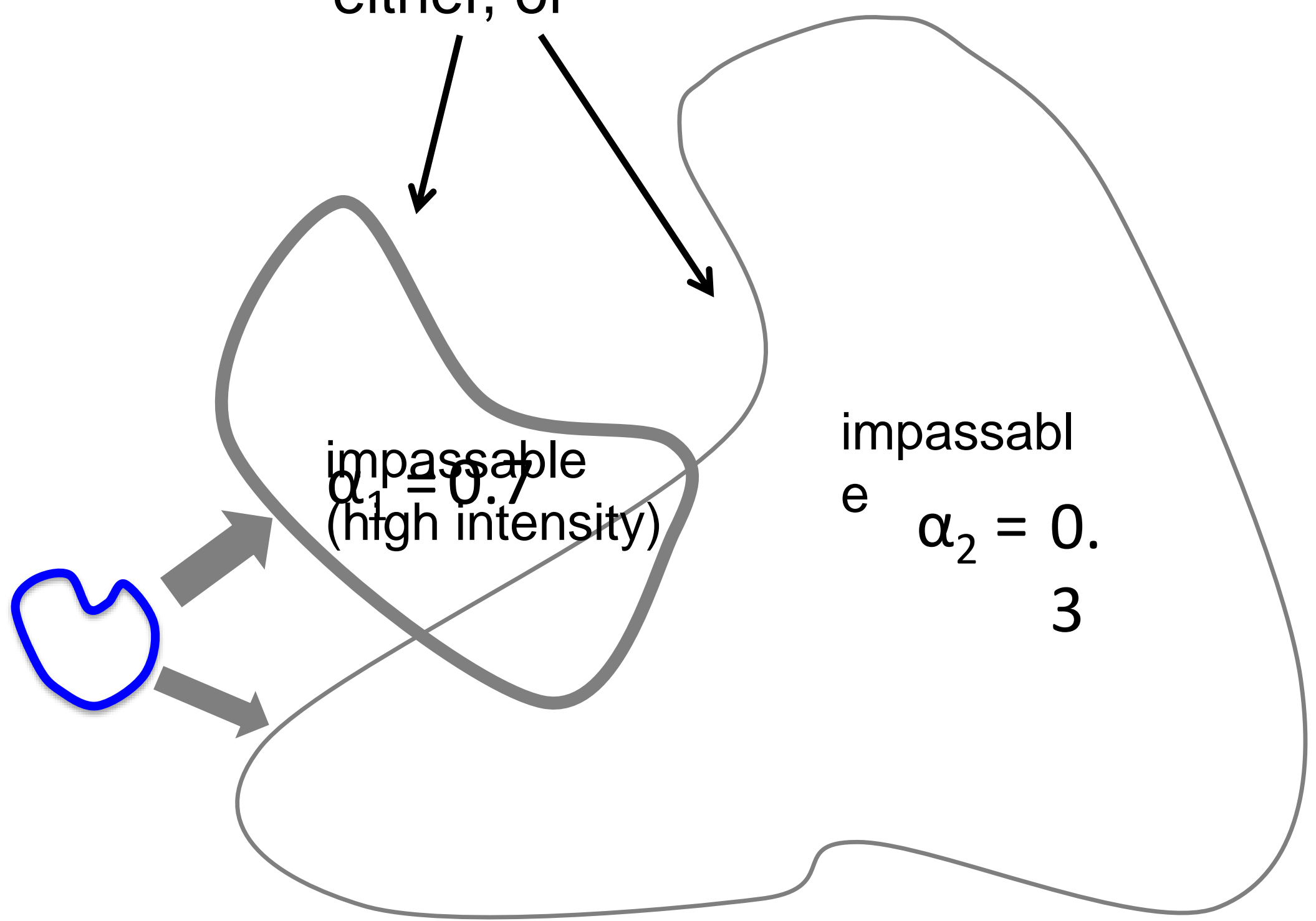




\section{Re-routing for Weather Avoidance}

NASA

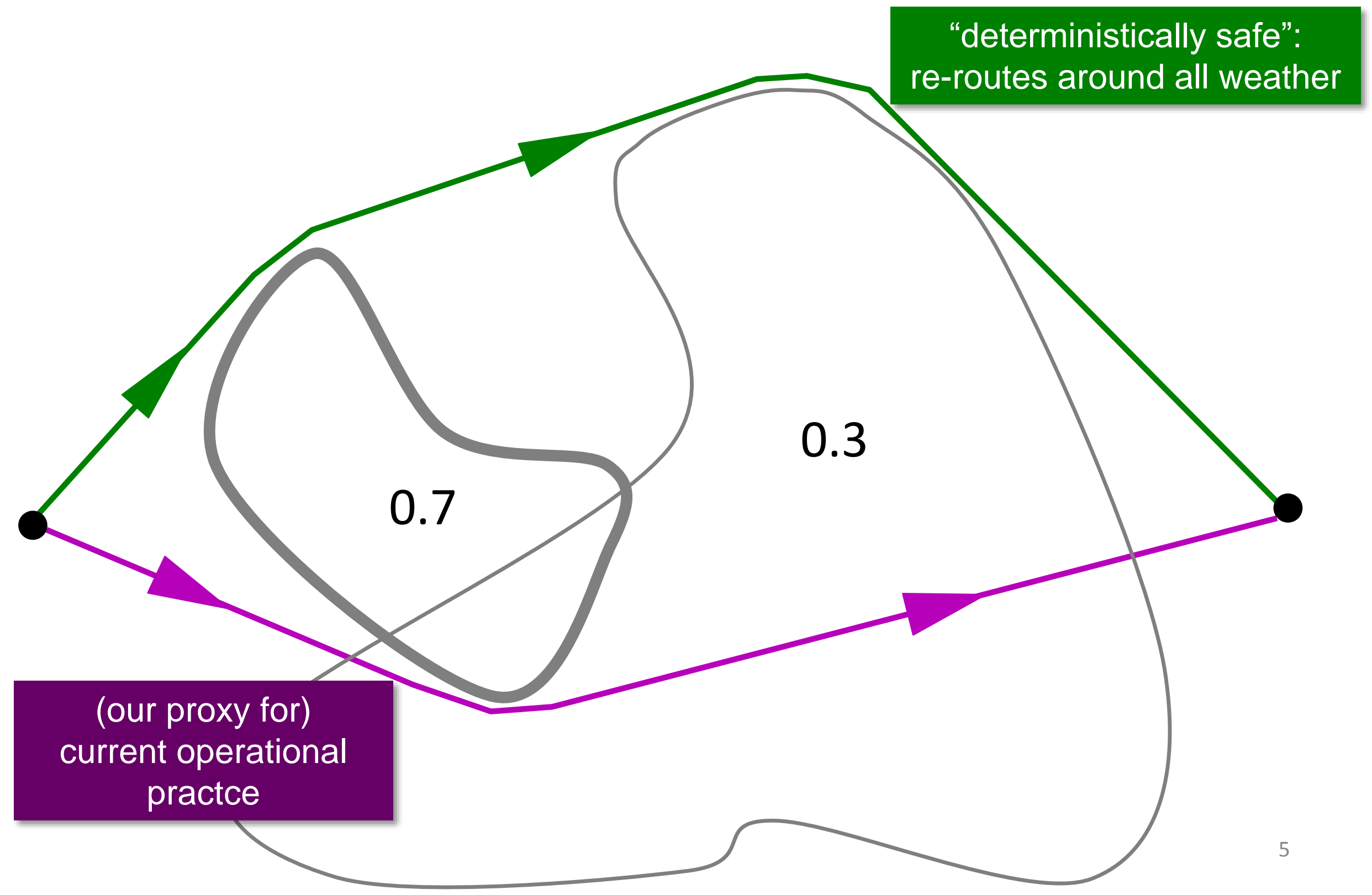




\section{Motivation for Risk-Hedging}

can incur high flight operation cost

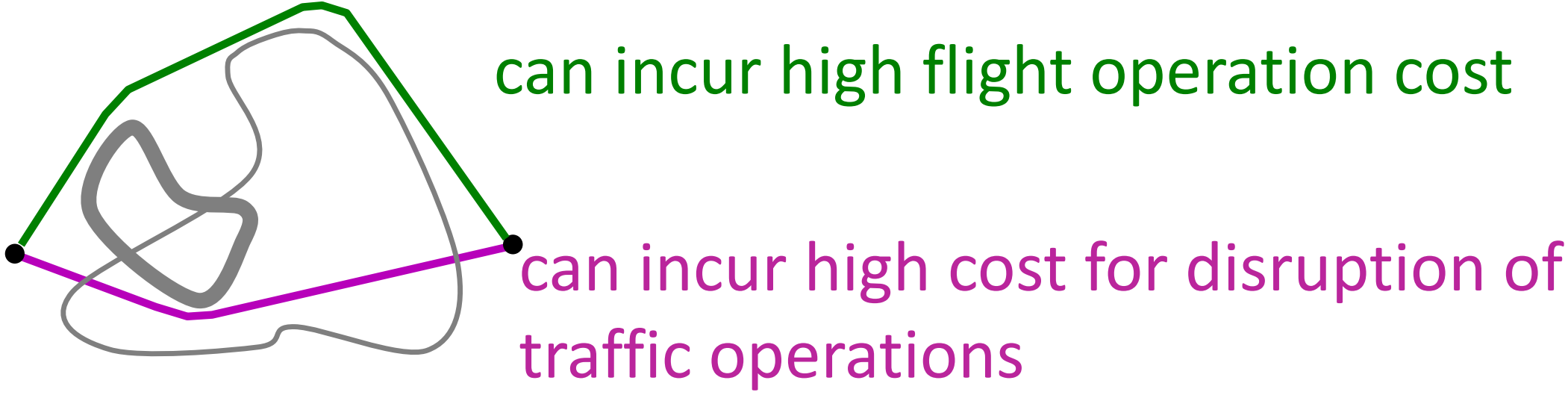

Risk-hedged approach:

minimize a combination of these two costs (later slide)

- "Risk" refers to risk of disruption caused by tactical re-routing; hence a path has high risk if a large segment lies within a weather instantiation of high likelihood

- Research is far term: assumes ensemble weather forecast with multiple (instantiations + likelihoods)

- CDM (Collaborative Decision Making) Convective Forecast Planning (CCFP) currently provides a rudimentary version of the desired capability 


\section{Example CCFP Advisory}

EXPERIMENTAL CDM CONVECTIVE FORECAST PLANNING GUIDANCE

VALID: 2109 UTC THU 19 MAY 2016

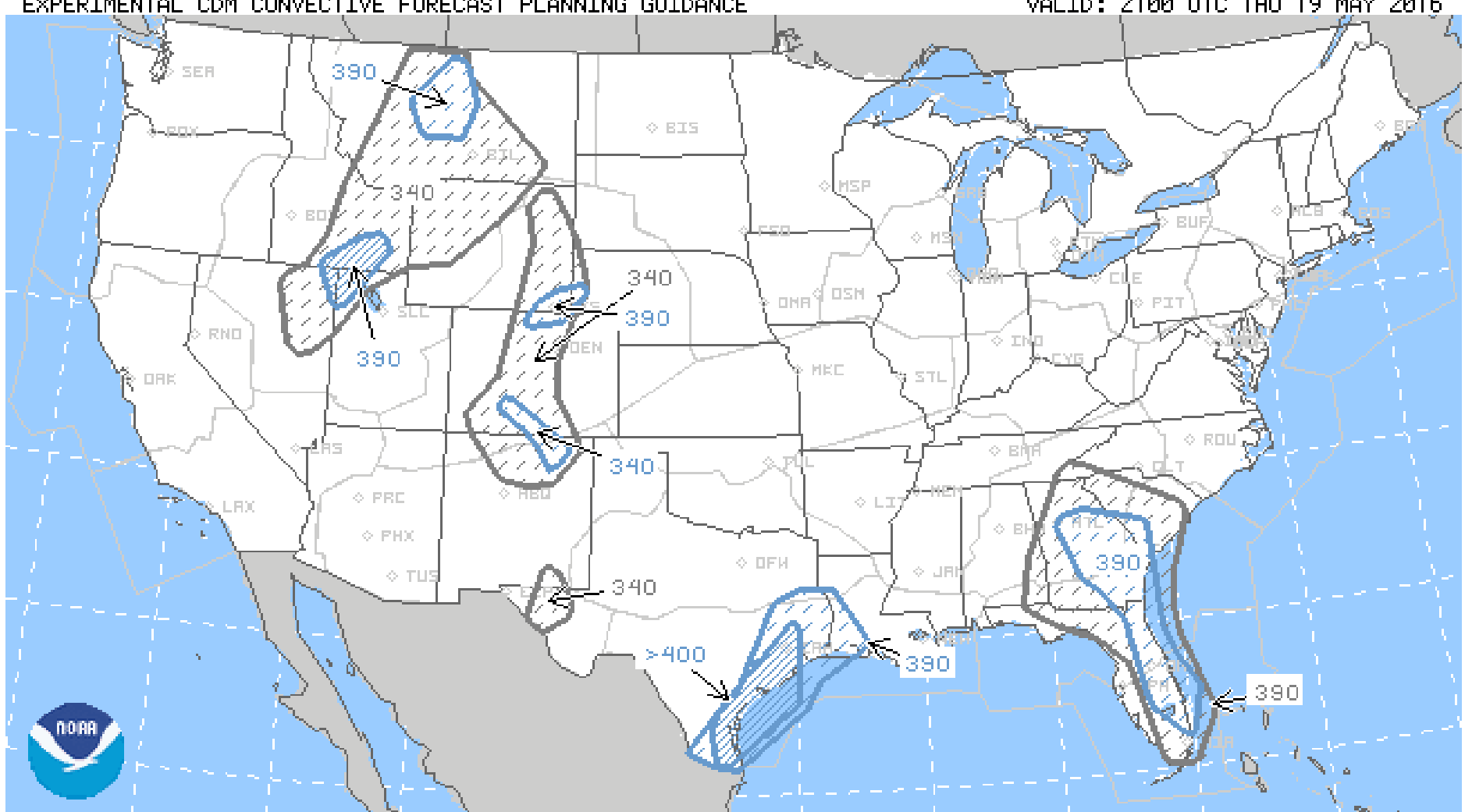

AVIATION WEATHER CENTER (NOAA/NWS/NCEP)

CONFIDENCE:

LOW HIGH

$25-49 \% \quad 50-100 \%$

CONYECTIVE 25-39\%

COVERAGE: MEDIUM+ $40-100 \%$
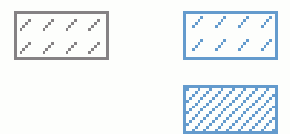

ISSUED: 1900 UTC THU 19 MAY 2016

\section{HEIGHT}

TOPS: 100 'S OF FEET MISL

25000 - 29000

30000 - 34000

35000 - 39000

390

$40000+$

$>400$ 


\section{Risk-Adjusted Field}

$$
\mathrm{P}=1 /\left(1-\Sigma \alpha_{i}\right)
$$

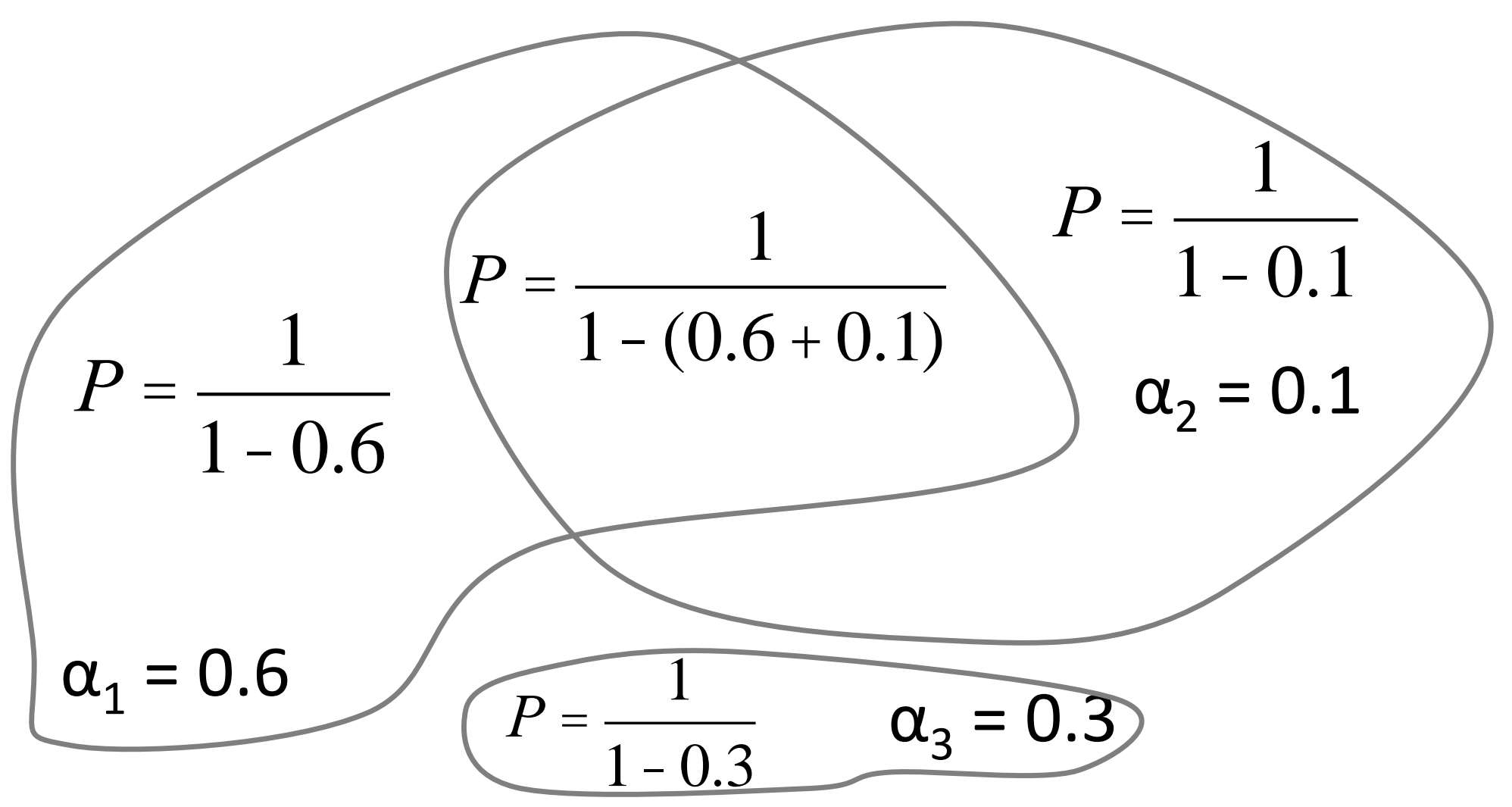




\section{Risk-Adjusted Field}

$$
\mathrm{P}=1 /\left(1-\Sigma \alpha_{i}\right)
$$

\section{$P=1.0$}

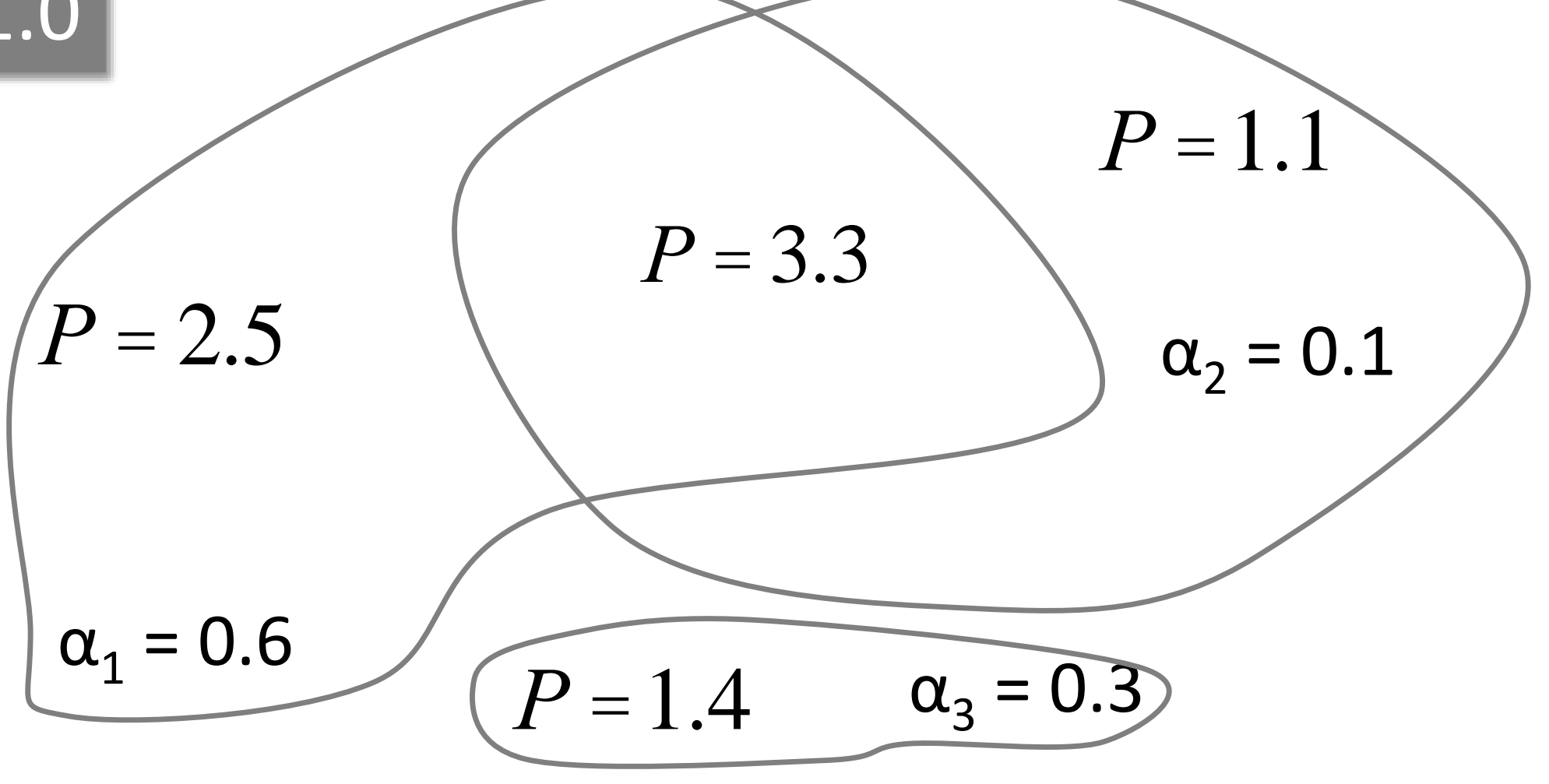




\section{Risk-Adjusted Path Length: the minimization objective}

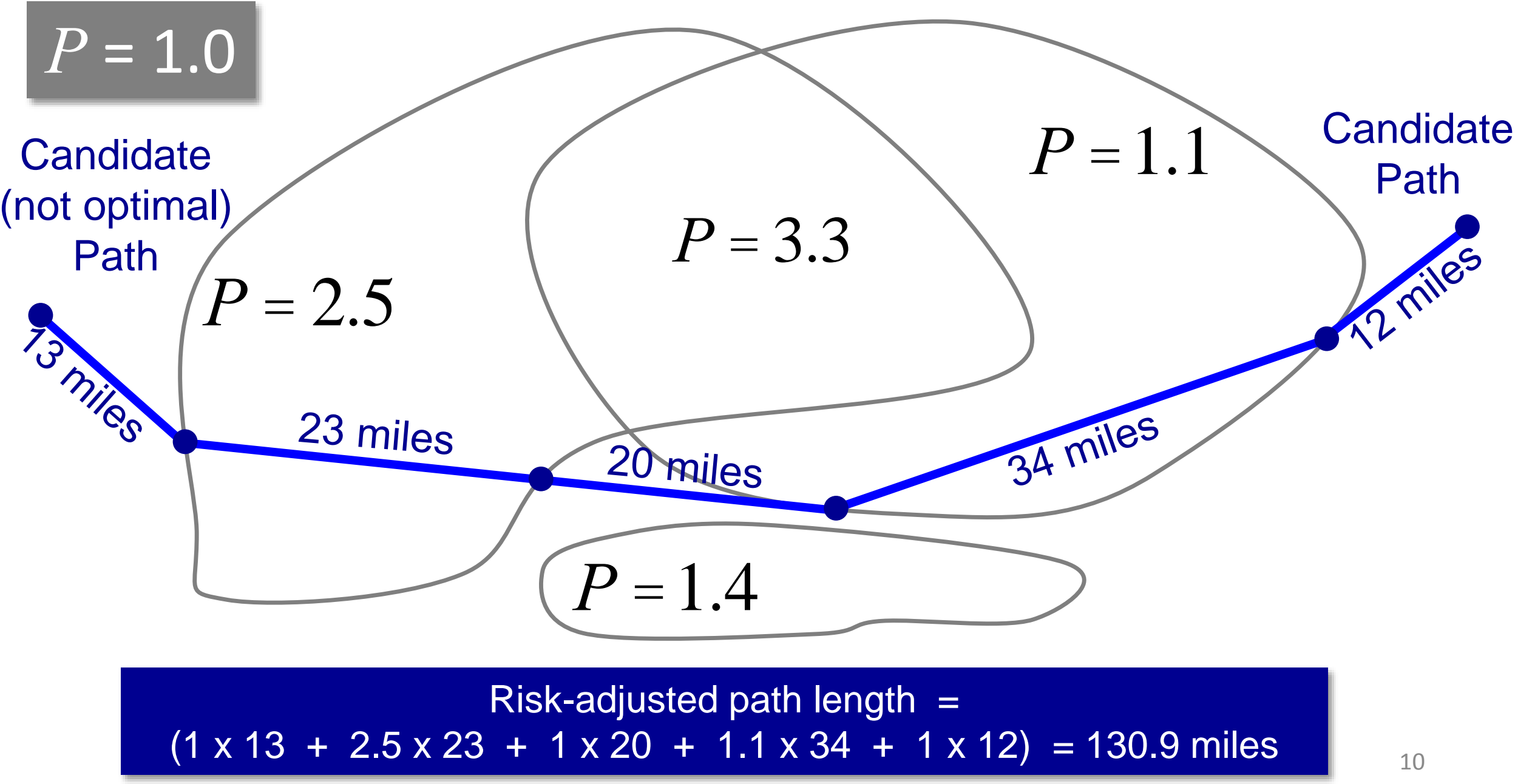




\section{Risk-Hedged Re-routing}

- Compute re-routes by minimizing risk-adjusted path length

- Evaluate the computed re-routing using these metrics:

- Path length (proxy for flight operation cost)

- Path risk (defined on next slide) 


\section{Metrics for Example \#1}

path

risk (nondim.)

0.20

0.15

0.10

riskhedged Less Path Risk

0.05

0.

0

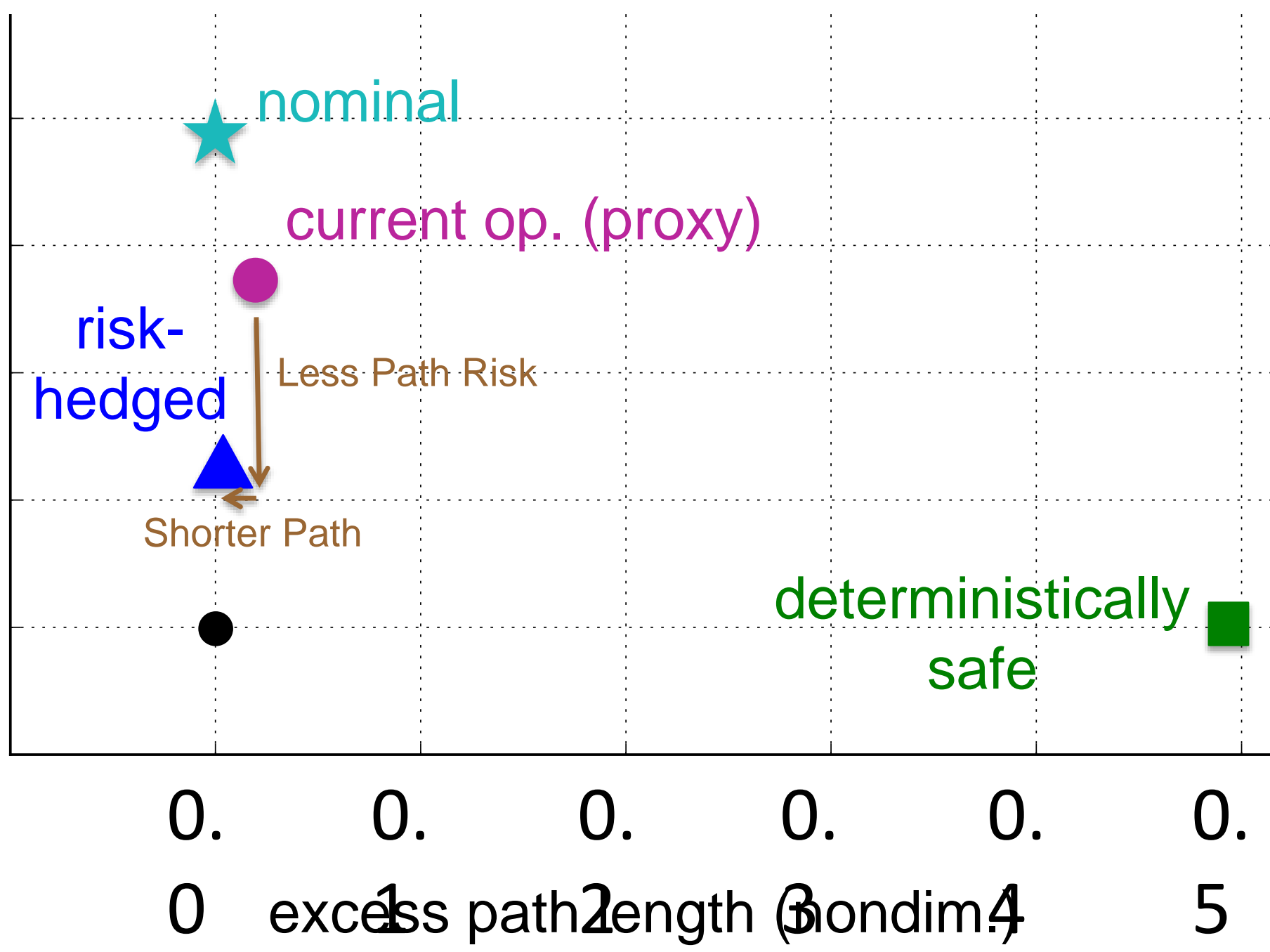




\section{Re-routing Options - Example \#2}

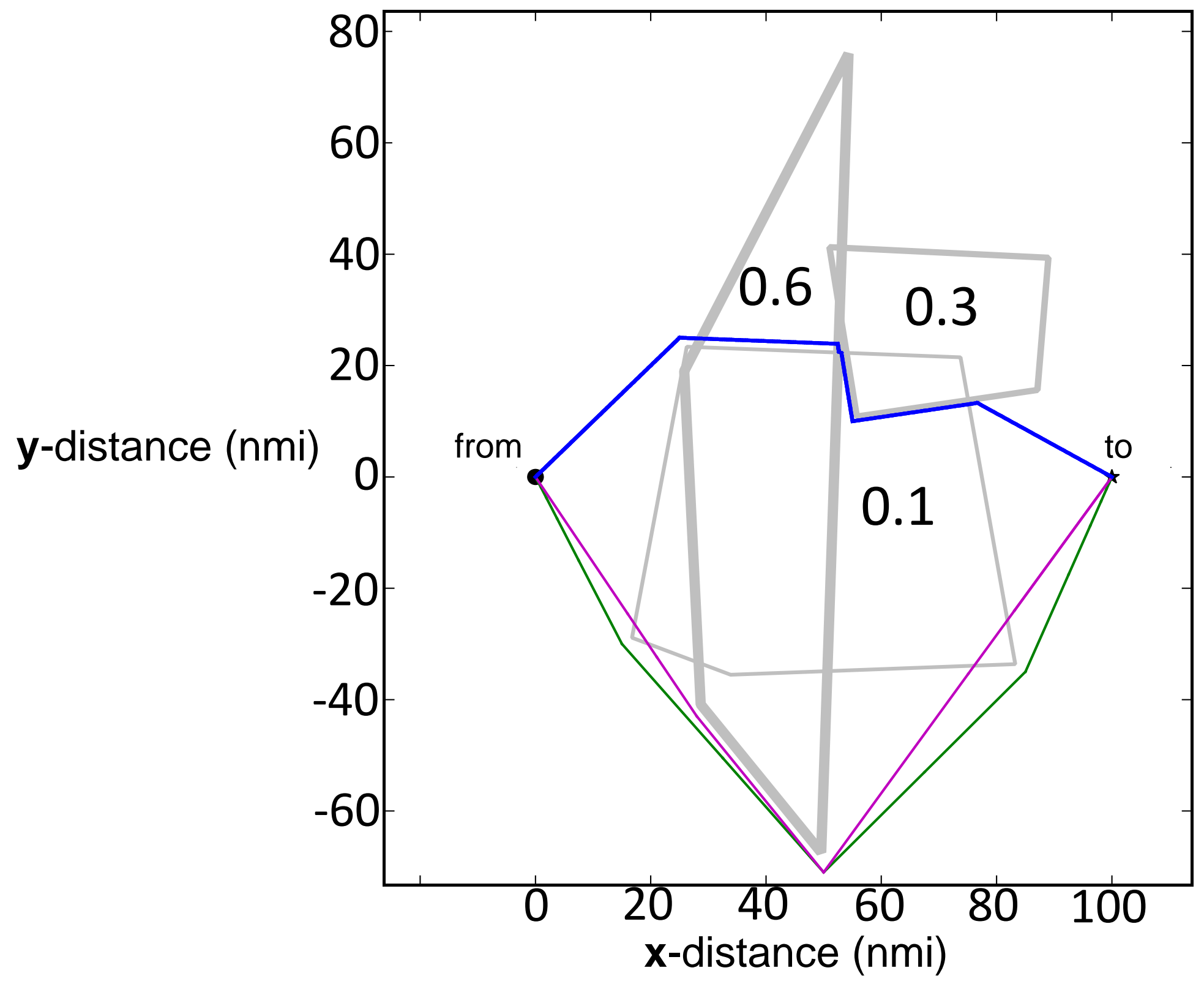


path

Metrics for Example \#2

risk (nondim.)

0.20

nominal risk-

0.15

0.10

Nore Path Risk

hedged

0.05

0.

0

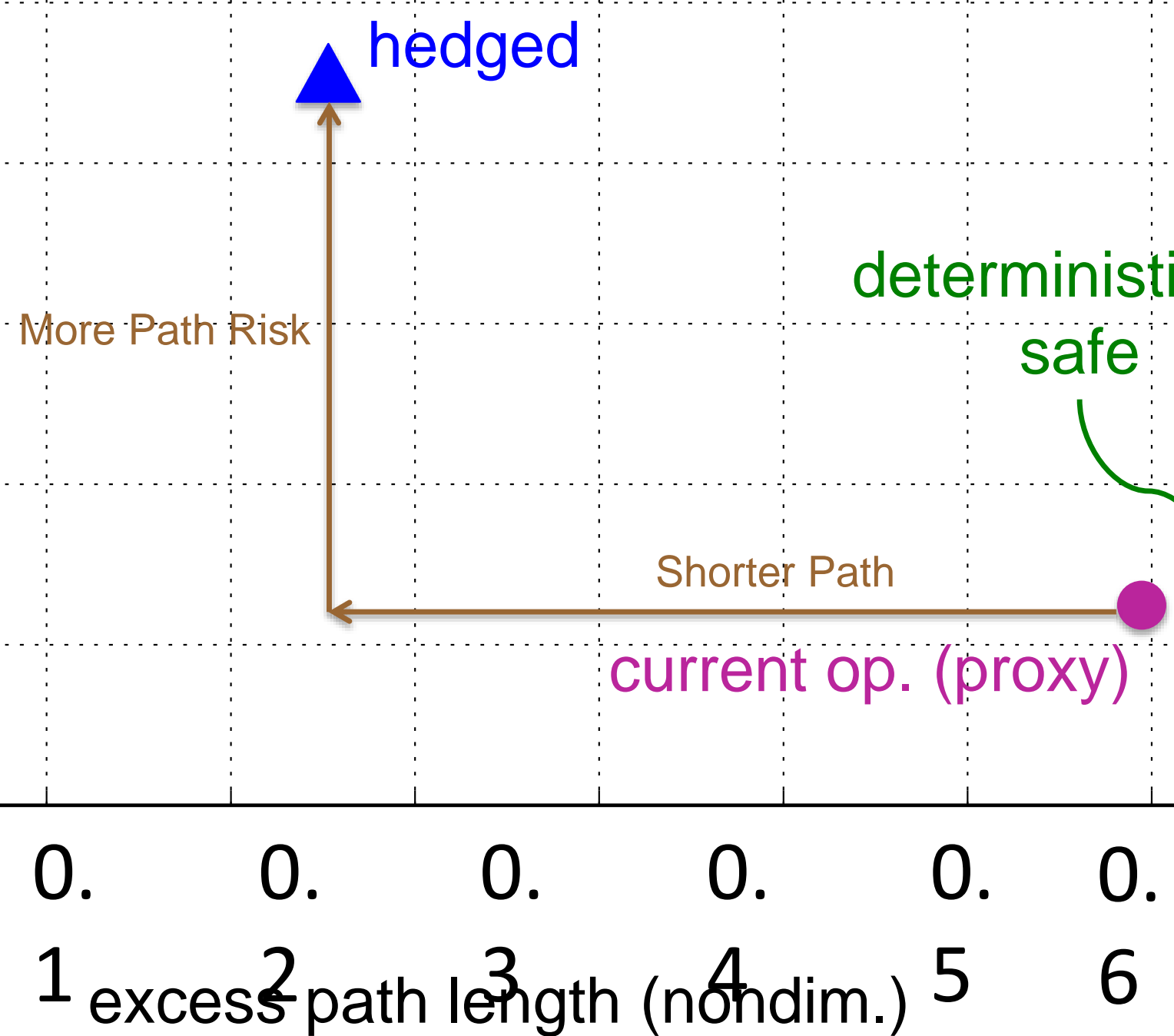




\section{Conclusion}

- In some weather avoidance scenarios, the risk-hedged re-routing is shorter and less risky than operational practice

- In other scenarios, risk-hedged re-routing can be:

- Less risky, but has a longer path

- More risky, but has a shorter path

- Potential application to re-routing for weather avoidance:

- Compute risk-hedged path

- Compare with operational-practice path for risk and path length

- Choose risk-hedged path if both safer and shorter 


\section{Backup Slides}




\section{Minimization problem: the Eikonal equation}

$\frac{1}{P(x)} \mid \operatorname{grad}(\min$. cost to endpoint from $x) \mid=1$ 


\section{Example Playbook Re-routing}

\section{Play: LEV EAST 1}

East-bound flows from ZLA, ZAB, ZFW, ZHU are merged and then split into two flows going to DC and NYC airports

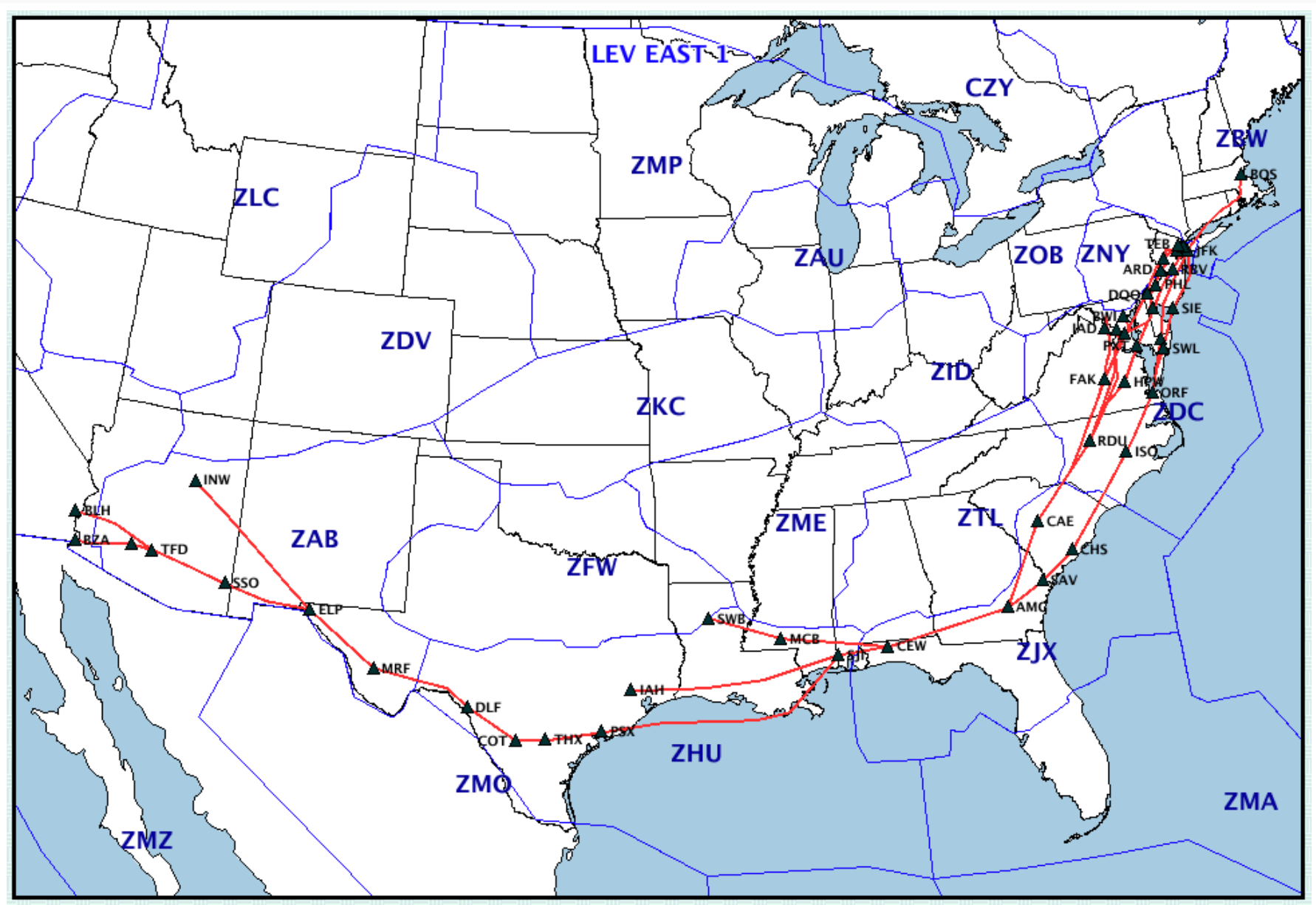

